\title{
Historia editorial de la traducción al inglés de Muertes de Perro en el archivo de la Fundación Francisco Ayala
}

\author{
History of the Translation into English of Muertes de \\ Perro in the Archive of the Fundación Francisco Ayala
}

\author{
Pablo Valdivia \\ University of Groningen
}

\section{RESUMEN}

Muertes de perro ha sido objeto de numerosos estudios críticos, pero hasta ahora no se había reconstruido documentalmente ni analizado pormenorizadamente la historia editorial de la traducción de esta novela al inglés. Este artículo, gracias a la abundante documentación presente en el Archivo de la Fundación Francisco Ayala, desconocida hasta este trabajo, explica cómo se desarrolló la presentación de Muertes de perro y de su autor en el contexto literario en lengua inglesa, al mismo tiempo que reflexiona sobre las implicaciones de la inmediata recepción de esta novela en el contexto internacional.

Palabras Clave: Francisco Ayala; Ilsa Barea; Muertes de perro; exilio.

\section{ABSTRACT}

Muertes de Perro has gathered the attention of several critical studies. However, so far, it was still missing a thorough analysis and reconstruction of the editorial history of its translation to English. This article, thanks to the existing documentation in the Archive of the Francisco Ayala's Foundation unknown until the present study, explains the presentation of Muertes de perro and its author in the English speaking literary context and reflects on the implications of its immediate reception at the international level.

Key words: Francisco Ayala; Ilsa Barea; Muertes de Perro; Exile Literature.

\section{INTRODUCCIÓN}

Muertes de Perro de Francisco Ayala ha sido considerada por parte de la crítica especializada como una de las obras narrativas más importantes del siglo $\mathrm{XX}$, sobre todo por la indagación que ofrece sobre las conflictivas relaciones

Copyright: (C) 2016 CSIC. Este es un artículo de acceso abierto distribuido bajo los términos de una licencia de uso y distribución Creative Commons Attribution (CC-by) España 3.0. 
entre poder e Historia. Junto a la lectura sociológica de la novela a la que en general estas aportaciones contribuyen, en lo que se refiere al estudio de Muertes de perro, la crítica también se ha ocupado de la técnica de su construcción y, particularmente, del perspectivismo narrativo en la obra. Desde una perspectiva más singular y con interesantes aportaciones, Muertes de perro también ha sido estudiada desde la óptica de la deconstrucción y desde los estudios de género.

Sin embargo, hasta este momento no se le ha prestado atención a la historia editorial del texto, no sólo en lo que se refiere a su redacción y publicación en el ámbito hispánico por la editorial Sudamericana en 1954, sino también en lo que atañe a su traducción al inglés y a la presentación del Ayala novelista en el ámbito editorial anglosajón (Ayala, 1964 y 1965). Y es este último aspecto el que por ello se convierte en el objeto principal de nuestro análisis en este artículo. El presente trabajo viene a cubrir ese hueco que hasta ahora no había sido estudiado en el caso de Muertes de perro, en parte debido a que la importante documentación que permite ocuparlo, de la que a continuación daremos pormenorizada cuenta, se ha conservado desconocida para los investigadores $\mathrm{y}$, por tanto, aún pendiente de análisis ${ }^{1}$.

\section{CORPUS DOCUMENTAL INICIAL}

Encontramos en el Archivo de la Fundación Francisco Ayala un conjunto documental formado por 36 cartas relacionadas con la traducción de Muertes de perro y copia de 7 reseñas publicadas en diversos medios sobre esta novela en el momento de su inmediata recepción en Estados Unidos y el Reino Unido. Todas estas cartas se encuentran mecanografiadas y entre ellas, además de la comunicación con los editores de Macmillan y Michael Joseph, con la traductora Joan MacLean o con los críticos Mildred Adams, Charles Poore y Carlos Clavería, sobre todo destaca la correspondencia entre Francisco Ayala e Ilsa ${ }^{2}$ Barea con un número total de cinco epístolas.

El primer documento que nos permite iniciar la reconstrucción de la historia editorial de la traducción de Muertes de perro al inglés es una carta con fecha de 19 de enero de 1959 del Departamento Comercial de la editorial Macmillan, firmada por el editor Emile Capouya, quien contesta a Ayala dándole las gracias por el envío de Muertes de perro para que fuera considerada para su traducción al inglés, pero explicándole que, tras un examen cuidado-

\footnotetext{
${ }^{1}$ Debo expresar mi agradecimiento al secretario del Patronato de la Fundación Rafael Juárez y a Manuel Gómez Ros, bibliotecario y documentalista del Archivo de la Fundación Francisco Ayala, por haberme permitido tener acceso a estos documentos y realizar este estudio.

${ }^{2}$ Nótese que en este trabajo hemos decidido mantener el nombre de Ilsa frente al de Ilse, ya que ella misma firma así en todas las cartas que hemos estudiado.
} 
so y «discussed it fully in our editorial meeting. However, we must advise you with regret that we do not feel we can make a publishing offer ${ }^{3}$. La carta se cierra con un párrafo final en el que Capouya cumple con las fórmulas de cortesía correspondientes a este tipo de comunicaciones formales deseándole éxito a Ayala en su intento de que la novela fuera traducida al inglés, al tiempo que le anuncia que «Your book is being returned to you herewith».

Este testimonio apunta a lo que bien hubiera podido ser la historia de un fracaso. Sin embargo, en una carta fechada un mes más tarde ${ }^{4}$, la editorial Macmillan volvía a ponerse en contacto con Ayala a través de la Senior Editor, Charlotte Painter, quien le informa del terrible error cometido por la editorial y le pide que por favor retomen la posibilidad de que la novela sea publicada por ellos en inglés: «Some weeks ago, you heard from my colleague, Mr. Capouya, about MUERTES DE PERRO, which we regretfully declined. We have since talked this over, and have heard from Professor Amaral about the book, and we feel that we may have made a mistake». Como muestra la cita, parece que la intervención del Professor Amaral, que el remitente da por conocido para Ayala, fue decisiva, ya que consiguió que la editorial volviera a interesarse por el libro. Efectivamente, el citado «Professor Amaral» ${ }^{5}$ era el profesor, de origen mexicano, José Vázquez Amaral, quien ocupó el puesto de director del Departamento de Español y Portugués entre 1947 y 1982 en la Universidad de Rutgers, donde Francisco Ayala trabajó desde 1958 a 1965.

Las respuestas de Ayala a estas cartas de Capouya y Painter no se encuentran en los archivos de la Fundación Ayala y, a no ser que hubieran sido guardadas en los archivos de la editorial Macmillan, no nos consta su conservación. No obstante, este hecho no impide la reconstrucción de la historia editorial de la traducción de Muertes de perro, ya que puede seguir rastreándose sin problemas a través del corpus documental que manejamos debido a la cita o a la referencia reiterada en algunas de las cartas restantes a los textos que no se han conservado.

El siguiente documento de la serie es una carta con fecha de 21 de julio de 1959. Hay un dato importante que no podemos dejar de lado. Mientras que las dos cartas anteriores fueron remitidas a Ayala a su dirección del 54 West 16th Street de New York, esta tercera de la editorial Macmillan tiene como destino la dirección de Ayala en la Facultad de Ciencias Sociales de la Universidad de Puerto Rico en Río Piedras. Ese hecho muestra que Ayala se encontraba, algo que también recoge en su libro Recuerdos y olvidos (1982), en un momento de transición entre Puerto Rico y Nueva York debido a su ocu-

\footnotetext{
${ }^{3}$ Carta de Emile Capouya a Francisco Ayala. Archivo de la Fundación Francisco Ayala, documento 167, 19 de enero de 1959.

${ }^{4}$ Carta de Charlotte Painter a Francisco Ayala. Archivo Fundación Francisco Ayala, documento 168, 18 de febrero de 1959.
} 
pación de un nuevo puesto como profesor universitario en Rutgers. Esta carta, la tercera de la serie, va firmada por Emile Capouya. En el documento Capouya transmite a Ayala las condiciones específicas del contrato que le proponían por la traducción de Muertes de perro. Las condiciones eran favorables, teniendo en cuenta el contexto del momento y el hecho de que Ayala era un autor desconocido en el ámbito literario anglosajón. Tales condiciones no sólo concretaban cifras y detalles relacionados con las ventas del libro, sino que también fijaban una propuesta con respecto a los derechos cinematográficos y el reparto de ganancias en caso de que la novela se llevara al cine. La oferta de la editorial para la traducción y publicación en inglés de Muertes de perro era una apuesta decidida que trasluce claramente la posibilidad de hacer de la novela un éxito. Los 500\$ de 1959 que se fijan como anticipo equivaldrían aproximadamente a unos 3.000 euros actuales, una cantidad más que razonable para un autor que estaba tratando de acceder al mercado estadounidense:

We should pay you, on the signing of the contract, an advance of $\$ 500$ against the following royalty scale: $10 \%$ of the cash received on the first 6000 copies; $10 \%$ of the book's list Price on the next $4000 ; 12 \frac{1 / 2}{\%}$ of the list price on the next 5000 copies, and $15 \%$ thereafter, except that if, in any fiscal year after the first year of publication, and after the sale of 6000 copies, sales fall below 1000 , royalty is to revert to $10 \%$ of the cash received for that year. (We interpret cash received to mean $60 \%$ of the list price of the book, since this figure represents the average net return form the bookseller, to whom we grant an average discount of $40 \%$ ) [...] If we were successful in negotiating for the sale of an English edition, we would divide proceeds with you 50/50, as we would in the case of the sale of second serialization rights. Should motion picture or similar rights be sold, we would obtain $10 \%$ of the proceeds. Translation and first serial rights would belong to you ${ }^{6}$.

Es cierto, como se aprecia en la cita, que la escala porcentual de regalías es singular ya que no toma como base el precio de venta al público de cada ejemplar, sino el precio del mayorista reducido incluso hasta un $60 \%$ para los 6000 primeros ejemplares, lo cual no supone un mal acuerdo. Además se le ofrece al autor un $50 \%$ por la subedición en inglés, algo que todavía hoy es un estándar de mercado que se sigue manteniendo. Por tanto, entre el rechazo inicial y la favorable propuesta que acabamos de leer la diferencia más que grande es esencial, por lo que, insistimos, la intervención del Profesor Amaral parece haber sido providencial. El cambio es tan radical que incluso Capouya pide a Ayala que «We should like to have an option on your next work, on terms to be arranged between us». La entrada de la primera novela de Ayala traducida al inglés no sólo había conseguido unas condiciones económicas

\footnotetext{
${ }^{5}$ Debo este dato a la erudición de Manuel Gómez Ros, bibliotecario de la Fundación Francisco Ayala.

${ }^{6}$ Carta de Emile Capouya a Francisco Ayala. Archivo Fundación Francisco Ayala, documento 160, 21 de julio de 1959.
} 
razonables ${ }^{7}$, sino que abría unas perspectivas verdaderamente esperanzadoras para consolidar la presencia de la obra del autor en el ámbito anglosajón.

A los pocos días, como ya anunciaba Capouya en su carta, Ayala recibió otra comunicación de la editorial Macmillan con fecha de 17 de agosto de $1959^{8}$ con destino, ahora sí, a la dirección de Ayala en Nueva York, en la que se le hace entrega del contrato firmado y del cheque de 500 dólares en concepto de anticipo. La carta está firmada por B. Crizzuti, secretaria de Emile Capouya. El acuerdo ya estaba cerrado.

\section{TRADUCCIÓN AL INGLÉS DE MUERTES DE PERRO}

Hasta aquí hemos dado cuenta de los documentos iniciales que conforman la historia editorial de la traducción de Muertes de perro al inglés. Tras la conclusión de los detalles contractuales, en principio esta novela de Ayala habría de ser rápidamente publicada en los Estados Unidos. Macmillan era una de las editoriales más fuertes del ámbito norteamericano y para Ayala todo ello supondría su presentación como novelista en el contexto literario en lengua inglesa. No obstante, aún quedaba por solventar un aspecto de considerable importancia que, con el tiempo, se convertiría en una verdadera pesadilla para Ayala: ¿quién se encargaría de la traducción de la novela?

Cuando por fin aparezca la novela en las ediciones en inglés publicadas en Estados Unidos y en el Reino Unido, tras los años y las vicisitudes de las que daremos cuenta, el nombre de la traductora que figura en ellas es el de Joan Maclean. Sin embargo, Ignacio Soldevila Durante, quien editó la correspondencia entre Francisco Ayala y Max Aub correspondiente a aquellos años, destacó en una de sus notas al pie de página que «es curioso que la traducción, publicada por MacMillan en Nueva York y en Londres, aparece como obra de Joan McLean, y no de Ilse Barea, como parecía suponerse de la anterior correspondencia [se refiere a la correspondencia Ayala-Aub donde hay alusiones a la traducción de Muertes de perro]. Se podría deducir que su actuación fue de intermediaria, sencillamente, a menos de que usase un nombre de pluma como traductora, cosa poco probable para una persona que tenía tanto prestigio como traductora» (Soldevila, 2001: 116). Los entresijos de la historia de la traducción de Muertes de perro son en realidad, como prueban los documentos que examinamos a continuación, mucho más complejos de lo que Ignacio Soldevila podía imaginar.

En la serie de documentos que hemos estudiado en la Fundación Francis-

\footnotetext{
${ }^{7}$ Debo expresar mi agradecimiento por el análisis cabal de los detalles contractuales y económicos de la traducción de Muertes de perro a Antonio Muñoz Vico.

${ }^{8}$ Carta de Barbara Grizzuti a Francisco Ayala. Archivo Fundación Francisco Ayala, documento 159, 17 de agosto de 1959.
} 
co Ayala encontramos una carta, que lleva fecha de 27 de septiembre de $1959^{9}$ y está dirigida a Ayala a su dirección ya señalada de Nueva York. Se trata de una carta mecanografiada con remite de Ilsa Barea y dirección del 10 de Lansdowne Crescent, London, W.11. En el documento Ilsa Barea agradece a Ayala una carta anterior, que no se conserva en los archivos de la Fundación Ayala, en la que nuestro novelista la invitaba a que se ocupara de la traducción de Muertes de perro al inglés. El que Ayala eligiera a Ilsa Barea para este trabajo se puede justificar por dos motivos: por un lado Ilsa Barea estaba reconocida en ese momento como una de las grandes traductoras del español en el ámbito inglés, por lo que se encontraba muy bien posicionada en el mundo editorial, tal y como Max Aub llega a expresar en su correspondencia paralela con Ayala «Madame Barea [...] sigue siendo la única que tiene vara alta en eso de las ediciones españolas en Inglaterra» (Soldevila, 2001 83); por otro lado, Ayala había mantenido una estrecha relación con Leopold Kulcsar, primer marido de Ilsa Barea, mientras vivió en Praga durante la Guerra Civil española cuando trabajaba para el Servicio de Inteligencia Militar en la Embajada de España bajo las órdenes de Jiménez de Asúa ${ }^{10}$, tal y como lo relata el propio autor en sus Recuerdos y olvidos (1982), algo a lo que alude la señora Barea en su carta:

Le debo mis sinceras gracias por su carta, tan cordial y halagüeña, y por su interesantísima novela. Me gusta, me gusta mucho; y espero — «I hope»- que Mr. Emile Capouya podrá consentir a mis condiciones, porque de todos los libros que recientemente he tenido que traducir, éste se destaca por una cualidad intelectual que me atrae de veras.[...] Me recuerdo bien de su CABEZA DE CORDERO, libro que mandó a Arturo hace años y que nos gustaba mucho a los dos, aunque me temo que Arturo, como de costumbre, nunca le dio las gracias. Tenemos, usted y yo, a unos amigos en común, sobre todo a José María Domínguez. Y ¿me equivoco si creo que usted ha conocido a mi primer marido en Praga, a Leopold Kulcsar? ${ }^{11}$.

Ilsa aceptó el encargo e hizo llegar a los editores de Macmillan las condiciones de su contrato. Ella manifestaba al final de esta carta que «haré mi mejor para su libro» y su deseo de que «esto sea el principio de una colaboración fructífera». Sin embargo, y ya lo adelantamos, Ilsa Barea jamás llegó a concluir la tarea comprometida, lo que retrasó, de manera alarmante y claramente perjudicial para los intereses de Ayala, la publicación de la traduc-

${ }^{9}$ Carta de Ilsa Barea a Francisco Ayala. Archivo Fundación Francisco Ayala, documento 155, 27 de septiembre de 1959.

${ }^{10}$ De Jiménez de Asúa se conserva un valioso informe en la Fundación Pablo Iglesias en el que relata la muerte de Leopold Kulcsar y donde especifica, de manera muy clara, cómo todo el servicio de espionaje de la República dependía de la estructura que Kulcsar había articulado en Centroeuropa.

${ }^{11}$ Carta de Ilsa Barea a Francisco Ayala. Archivo Fundación Francisco Ayala, documento 155, 27 de septiembre de 1959. 
ción de Muertes de perro. En este aspecto, los documentos prueban que ni Ilse Barea fue una intermediaria ni el nombre de Joan McLean respondía a un nombre de pluma, al contrario de lo que Soldevila señalaba. Los acontecimientos de la traducción fueron otros.

Efectivamente, a esta carta dirigida a Ayala con fecha de 27 de septiembre de 1959, Barea adjuntó para nuestro autor otra que contenía su respuesta al editor Emile Capouya de Macmillan: «Le envío la copia de la carta que mando al Sr. Capouya, para evitar una pérdida de tiempo».

Por esta copia conocemos que Ayala había escrito a Ilsa Barea con fecha de 21 de septiembre de 1959 proponiéndole el proyecto de traducción de Muertes de perro, carta de la que, insistimos, no se halla copia en el archivo de la Fundación. En su misiva a Capouya, Barea explica que la traducción de la novela de Ayala supone una gran dificultad técnica y que por ello calcula que completarla le llevará al menos cuatro meses, ya que no puede dejar de lado otros compromisos de trabajo: «I calculate that the actual translation will take me all of four months, since I would never be disastrous for me. In brief, if you still wish to entrust this translation to me, I could commit myself to no earlier date for the delivery of the MS. fit for the printers than the last of April [...] If your production program were to permit ampler time, say, the end of May or middle of June, I would be delighted» ${ }^{12}$.

Como podemos comprobar en este fragmento, Ilsa Barea deja muy claro que como mucho entregaría el trabajo final en el plazo correspondiente a un periodo de siete a nueve meses. Como condiciones de pago sugiere lo que denomina la «U.N. scale», que equivale a «which is twelve dollars a thousand words, with half of the computed fee payable on agreement or the signing of a contract, and half on delivery of the complete translation». Y añade «This is my invariable arrangement with British publishers», lo cual correspondía, dentro de su contexto, a unos honorarios más que razonables.

En el documento 152 del archivo de la Fundación Ayala, encontramos la siguiente carta del conjunto de las que, remitidas por nuestro autor a la traductora, sí se conservan. Lleva fecha del 3 de octubre de $1959^{13}$. En el documento queda claro que la apuesta de Ayala por Ilsa Barea como traductora de la novela es completamente firme: «trataré de hablar con Capouya, con objeto de convencerlo, si tiene dudas respeto de la tarifa que usted le menciona, de que vale la pena tener una traducción como sólo usted es capaz de hacerla. Creo que ésa sería la única objeción, dado que el plazo establecido por usted es prudencial y se tiene la seguridad de que habrá de cumplirlo». Y además muestra la lucidez de Ayala al anticipar lo que supondría la recepción de

\footnotetext{
${ }^{12}$ Carta de Ilsa Barea a Emile Capouya. Archivo Fundación Francisco Ayala, documento 154,27 de septiembre de 1959 .

${ }^{13}$ Carta de Francisco Ayala a Ilsa Barea. Archivo Fundación Francisco Ayala, documento 152, 3 de octubre de 1959.
} 
Muertes de perro en el ámbito anglosajón, ya que afirma que «Supongo que la editorial querrá hacer del libro un éxito (y en cierto modo, ésa es la prisa, pues parece que el momento es oportuno, dadas las circunstancias hispanoamericanas) razón de más para que paguen decentemente la traducción». Y ya declara además que «Y si, en efecto, llegara a venderse bien, sería cuestión, seguramente, de traducir algunas de mis otras cosas, como LA CABEZA DEL CORDERO, que usted menciona tan amablemente». Por tanto podemos afirmar aquí que Ayala era plenamente consciente de la importancia de esta traducción para su presentación editorial en el mercado en inglés, expectativa que podía verse influida por el clima de inestabilidad y de crisis política que cruzaba el Centro y el Sur de América. Así se lo hace saber a Ilsa Barea, en la que confía plenamente para que esta empresa culminara en tiempo y forma. Se trataba de una oportunidad excelente para la consagración de Ayala como novelista en el ámbito internacional e Ilsa Barea podía contribuir decisivamente a ello si aceptaba la traducción de sus obras. De ahí que en un momento de la carta Ayala aluda directamente al ex-marido de Barea en los siguientes términos:

En efecto, conocí a Leopold Kulcsar, fui amigo y asistí a su muerte. Era un hombre excelente, y siempre nos habló de usted con respeto y entusiasmo: inclusive nos contó la entrevista con usted, tal cual luego había de relatarla Arturo en su libro. En cierto modo, mi amistad hacia usted pasa a través del recuerdo de aquellos años inolvidables y sin embargo, tan amargos de recordar.

A lo que añade:

Dígame — suponiendo siempre que el trato se cierre con Macmillan - si quiere que le remita otras cosas mías, y si le conviene recibir, o no, algunos de los comentarios que se han escrito sobre MUERTES DE PERRO.

Por tanto, como explica el documento 152, Ayala sabía que, de tener éxito la traducción de Muertes de perro, sus libros podrían alcanzar una proyección que sobrepasaría los límites del mercado en lengua española.

En efecto, en la siguiente carta, fechada el 3 de noviembre de 1959, Ilsa Barea comunica a Francisco Ayala que ya tiene el contrato firmado y que Capouya le ha ofrecido un «plazo muy generoso» para completar la traducción:

Hace ya más de una semana recibí una carta del Sr. Capouya con el contrato, que devolví en seguida. Me han dado un plazo muy generoso para completar la traducción, y todo se queda arreglado; falta nada más que el cheque, que prometieron para cuando recibieran el contrato firmado por mí -y lo más importante, lo único importante, que es el trabajo mismo. Tengo mucho ánimo para ello y me siento algo impaciente, por tener que terminar tantas cosas antes ${ }^{14}$.

${ }^{14}$ Carta de Ilsa Barea a Francisco Ayala. Archivo Fundación Francisco Ayala, documento 156, 3 de noviembre de 1959. 
Nos resulta imposible determinar a qué se refería exactamente Ilsa Barea cuando hablaba de un «plazo muy generoso». No se conserva en el archivo de la Fundación ningún documento que concrete este aspecto. Tampoco hemos dispuesto del contrato de esta traducción y no creemos que sea posible, a no ser que se acabe encontrando en el archivo de la biblioteca Bodleian de Oxford a donde nos consta que, en un futuro quizás próximo, será enviada la documentación que conservaban los familiares de los Barea y que todavía no ha sido estudiada. Sin embargo, teniendo en cuenta la información presente en las cartas de las que venimos dando noticia, cabe suponer que como mucho Capouya debió concederle el máximo lapso temporal que Barea había mencionado a Ayala, es decir, de siete a nueve meses.

En esta carta del 3 de noviembre, Barea además menciona algunos problemas de salud que le habían impedido escribir a Ayala antes: según ella tenía los dedos índice y corazón de la mano derecha «un poquillo artríticos (cosa con la que me dejó un encarcelamiento en el año pum, es decir en 1933...)». Por ello declara que ahora realiza su trabajo de traductora con una máquina de dictar «de esas de cinta, y con la ayuda de una muchacha que luego lo copia y recopia», artilugio que ha comprado con el cheque del anticipo por la traducción de Muertes de perro. La carta termina con una serie de alusiones a conferencias y proyectos personales de Ilsa y a lo que ella llama su «ilusión [de] que entre el autor y el traductor debe existir una comprensión mutua - si no es una traducción de "pan y mantequilla", como la que estoy terminando y otra que me está esperando».

Queda claro, por tanto, que esta traducción de Muertes de perro no era «una más» de las de «pan y mantequilla» de las que habla Ilsa, sino que ella parece también plenamente consciente de las cualidades y la importancia que tenía esta obra dentro del ámbito hispánico, así como de la necesidad de darse cierta prisa en completar el trabajo, ya que suponía la posibilidad de que Ayala pudiera seguir viendo sus obras publicadas en inglés y entrara en el mercado editorial norteamericano.

Insistimos una vez más: esta última comunicación se producía el 3 de noviembre de 1959. A partir de ese momento Ilsa Barea disponía de siete a nueve meses para completar la traducción. Sin embargo, inexplicablemente, se produjo un largo silencio. La siguiente carta de la serie la encontramos en el documento signado con el número 157, firmada por Ilsa Barea, dirigida a Ayala y fechada el 22 de julio de 1961.

Entre la carta de 1959 y la de 1961 distan 21 meses. Por la carta del 22 de julio de 1961 se entiende que Ayala escribió en numerosas ocasiones a Ilsa Barea alarmado ante el claro retraso que estaba sufriendo la traducción de Muertes de perro, pero ella no contestó hasta la carta que citamos del 22 de julio, cuyo remite proviene ya de otra dirección: 26 Upper Park Road London, N.W.3. En este documento Barea, a modo de justificación, afirma que: 
Mi distinguido amigo:

Espero que me deje llamarle así, a pesar de su comprensible e inevitable irritación y rabia conmigo...

Primero: lo que Salazar Chapela me indica que Vd cree, a causa de THE HORNS OF FEAR, no es así. En esto, al menos, soy completamente inocente. Faber \& Faber de Londres me encargaron con la traducción de la novela de A. M. de Lera unos seis meses o más antes de que Vd se puso en contacto conmigo, y terminé la traducción al principio del año pasado.

Además Barea indica que esta obra, The horns of fear, fue un «bread and butter work» y que si la tuvo que terminar antes que Muertes de perro se debió a que había sido comprometida con anterioridad. Ilsa reconoce que no ha cumplido con el compromiso adquirido con Ayala y con la editorial, como demuestra al afirmar que «Ya tiene bastantes razones para quejarse de mí, pero eso [se refiere a la supuesta acusación de que ella se hubiera ocupado de encargos posteriores a Muertes de perro dándoles prioridad frente a la obra de Ayala] sería un falta de lealtad absoluta, cosa de la que no creo ser capaz».

Por otro lado, llama la atención que en todas las cartas Ilsa Barea cometa una y otra vez el error de denominar la novela como Muertes de perros en plural y no en singular. El error es sistemático y, como mínimo, da que pensar sobre el supuesto rigor y el perfeccionismo, sobre la profesionalidad responsable que Ilsa quería esgrimir como justificación de su retraso en el trabajo, o sea, de su flagrante incumplimiento. Los otros motivos a los que alude tienen estrictamente que ver con su salud. Barea declara que sufrió dos colapsos como consecuencia de una combinación de estrés y de su condición de diabética. Sin embargo, la propia Ilsa reconoce que esas causas de ninguna manera excusan su silencio, el cual trata de justificar en los siguientes términos:

Me dirá por qué no le he escrito sobre todo esto antes. La contestación es simple. No quería dar excusas y más excusas, sino decir: he hecho esto, aquí está. Creía haber llegado al punto hace unos seis meses, pero no me gustó el resultado de mis trabajos tantas veces interrumpidos, y otra vez lo rehíce todo. El experimento de usar el dictáfono salió mal, porque para ello los problemas del estilo de MUERTES DE PERROS («A Dog's Death», ya que el plural es imposible en inglés) son demasiado sutiles. Tuve que escribirlo por máquina, yo misma; aquí entró otra dificultad... los dolores artríticos en dos dedos de mi mano derecha y a veces en el brazo entero, que se empeoran rápidamente cuando escribo mucho ${ }^{15}$.

Y la carta finaliza con una afirmación rotunda por parte de Barea en la que plantea que la traducción está a punto de ser concluida y sugiere a Ayala que un poco más de espera tendrá un resultado satisfactorio: «Creo sinceramente que el resultado final, que ya está en vista — aún si la última tarea de corrección y de copiar no está completamente terminada- justificará la enorme y desesperante espera».

${ }^{15}$ Carta de Ilsa Barea a Francisco Ayala. Archivo Fundación Francisco Ayala, documento 157, 22 de julio de 1961. 
La reacción de Francisco Ayala fue muy cortés y comprensiva a pesar de su justificado enfado que no sólo admite Ilsa Barea, sino del que dan cuenta varias alusiones en la correspondencia paralela que Ayala mantenía con Aub (Soldevila, 2001: 52-117). Hay que tener en cuenta que Ayala llevaba esperando ya casi dos años la traducción de Ilsa Barea. En el documento 158 del Archivo de la Fundación Ayala, que consiste en una carta con fecha del 5 de agosto de 1961, Ayala deja bien claro que su único deseo es que el trabajo se dé por concluido. Según explica en la carta, Ayala, además de las numerosas comunicaciones que le envió a Ilsa Barea y de las que no obtuvo respuesta, viajó a Inglaterra algún tiempo antes, durante ese verano de 1961, para intentar, entre otras cuestiones, encontrarse personalmente con Ilsa. De ahí que señale que:

Pero, en fin, ya éste se encuentra, como usted me dice, prácticamente concluido, y no tiene objeto volver sobre el pasado. Lo que sí le he de rogar a usted ahora es que haga llegar a Macmillan sin más demora el original, para que al comenzar septiembre, tras las vacaciones estivales, puedan ellos disponer por fin su publicación. Estoy seguro de que su trabajo habrá sido tan brillante como cuantos salen de sus manos, y de que, según me dice al final de su carta, habrá que dar por bien empleada después de todo «la enorme y desesperante espera» ${ }^{16}$.

A lo que prosigue insistiendo en el hecho de que el tiempo apremia y en la importancia de que la traducción se encuentre en Nueva York para el 1 de septiembre, ya que Macmillan y otras editoriales «esperan ver la reacción que suscita en el ambiente para emprender, si es favorable, la publicación de otras obras mías, entre ellas una nueva novela, ligada a MUERTES DE PERRO, que ya debe estar imprimiéndose en la Argentina».

Por tanto, el carácter decisivo que una publicación de estas características por Macmillan podía tener en la carrera literaria de Ayala era tan evidente como para que él volviera a reiterarlo a Ilsa Barea con el objeto de que tomara conciencia de hasta qué grado le estaban perjudicando esos retrasos. A pesar de los esfuerzos ponderados de Ayala, esta será la última carta de la serie entre el autor y la traductora. No se conservan nuevos intercambios de cartas en el Archivo de la Fundación Ayala y mucho nos tememos que no debieron existir ya que, según traslucen documentos posteriores ${ }^{17}$, ante los continuos retrasos de Barea en la entrega del trabajo, la relación epistolar y personal debió de darse por concluida (Soldevila, 2001: 111).

El 22 de julio de 1961, Ilsa Barea había asegurado a Ayala que tenía la traducción prácticamente concluida. No obstante, en una carta del 4 de mayo

${ }^{16}$ Carta de Francisco Ayala a Ilsa Barea. Archivo Fundación Francisco Ayala, documento 158, 5 de agosto de 1961.

${ }^{17}$ En una carta dirigida a Aub de octubre de 1961 la califica como «sinvergüenza» y en otra carta, también a Aub y ya del año 1964, como «cerda». Consideramos que, después de tantos años, el enfado de Ayala estaba perfectamente justificado y que por tanto estos calificativos no contradicen ni el tono educado ni la paciencia que Ayala demostró. 
de 1962, casi 11 meses más tarde, enviada por la editorial Michael Joseph a A. L. Hart de la editorial Macmillan, leemos que Ilsa Barea no sólo se encontraba lejos de dar remate a la traducción, sino que le había dado prioridad a una novela de García Hortelano que había sido galardonada con el premio Formentor. Macmillan había contratado una subedición con Michael Joseph. Ilsa Barea, por ello, estaba en contacto con los editores de las dos compañías porque su traducción habría de ser la que se lanzara al mercado en los dos ámbitos editoriales.

Definitivamente queda claro que lo que en un principio comenzó con un buen acuerdo, que aseguraba una fiable y pronta traducción de Muertes de perro, se había tornado en una carrera de obstáculos. Ilsa Barea, sin duda por la insistencia de Francisco Ayala ante las editoriales, justamente preocupado por los retrasos en la traducción de la novela, respondió en una carta de la que la editorial Michael Joseph reproduce algunos fragmentos en la citada comunicación que establece con los editores de Macmillan. Y su principal excusa fue la de que los retrasos se debían a que su trabajo con la novela de García Hortelano había tenido que recibir prioridad frente al de la de Ayala, apuntando además la facilidad de la traducción de la novela de García Hortelano frente a la complejidad de Muertes de perro. Además, otra vez, Ilsa Barea vuelve a alegar problemas de salud como justificación de su incumplimiento de los plazos de la entrega de la traducción de la novela de Ayala y reitera su dificultad para conseguir una traducción satisfactoria. Según ella: «As I said, I shall force myself now to put a stop to my endless revisions, and perhaps, this will leave me with a less strained and unhappy conscience than I have now» ${ }^{18}$.

Lo que resulta más sorprendente es que el editor de Michael Joseph, que no firma la carta, asume con normalidad las excusas presentadas por Ilsa Barea a pesar de que ésta llevara más de dos años y medio de retraso sobre la fecha que ella misma acordó para la entrega de la traducción. Sin embargo, Emile Capouya, que para el año 1962 ya era Senior Editor de Macmillan, envió a Ayala una copia de la carta de la editorial Michael Joseph que mencionamos. La copia acompañaba a una carta del propio Capouya en la que afirmaba: «I note that she still has not committed herself to a date, and I shall try to get het to do so again. Messrs. Michael Joseph appear to be satisfied with her explanation, but I must confess that I am not» ${ }^{19}$. Efectivamente, Capouya, mucho más avispado que el editor inglés, se da cuenta de que Barea ni siquiera ha concretado una fecha de entrega.

Es en ese momento cuando Emile Capouya decide intervenir definitivamente en el asunto y en una carta del 23 de abril de 1962 escribe a Ayala expli-

\footnotetext{
${ }^{18}$ Carta de la editorial Michael Joseph Ltd. a Mr. Hart. Archivo Fundación Francisco Ayala, documento 148, 4 de mayo de 1962, p. 3.

${ }^{19}$ Carta de Emile Capouya a Francisco Ayala. Archivo Fundación Francisco Ayala, documento 149, 25 de mayo de 1962.
} 
cándole que ha descubierto que Ilsa Barea ha mentido, ya que en ningún caso les informó de la traducción de ese libro de Hortelano, pero que, aún peor, el premio Formentor fue otorgado a ese autor un año más tarde de que ella se comprometiera a traducir Muertes de perro: «I had not seen the notice of Mrs. Barea's translation of Hortelano. I am furious. The Prix Formentor that Hortelano was awarded post-dates, by at least a year, Mrs. Barea's commitment to translate Muertes de perro» ${ }^{20}$.

Para Capouya las razones aducidas por Barea son inexcusables y comunica a Ayala que va a tratar de conseguir el manuscrito de lo que Ilsa haya traducido ya, esté en el estado que esté: «When we have examined it, we shall know exactly where we stand and what to do next».

Entre abril de 1962 y el 18 de octubre de ese mismo año, debieron sucederse encuentros y reuniones personales entre Ayala y Capouya que finalmente condujeron a la búsqueda de un nuevo traductor. En una carta del 18 de octubre de 1962, Capouya indica a Ayala que ya se ha puesto en contacto con Anthea Joseph, de Michael Joseph Ltd., y que han acordado que Macmillan se encargará de encontrar un nuevo traductor ante la duda de que siquiera existiese el manuscrito de la traducción de Muertes de perro: «we mean to proceed with a new translation at once, except in the most unlikely event that the Barea manuscript actually exists and can be put into our hands» ${ }^{21}$. A lo que prosigue pidiéndole a Ayala que proponga él mismo un nuevo traductor.

Sin embargo, surgirá un nuevo obstáculo en esta historia de desafortunados acontecimientos. Por razones que nos son desconocidas, el editor Emile Capouya abandona la editorial Macmillan y no será hasta el 25 de febrero de 1963 cuando otro editor de Macmillan, Herbert Weinstock, retome el asunto de la traducción de Muertes de perro. Si tenemos en cuenta que desde el 27 de septiembre de 1959 Ilsa Barea ya tenía firmado un contrato con Macmillan para la traducción de Muertes de perro, la espera había sido tan exageradamente larga que la paciencia de Ayala ya había alcanzado tintes épicos. Hacía tres años y seis meses del encargo de traducción firmado por Ilsa Barea para un trabajo que se había comprometido a realizar en un plazo de siete a nueve meses.

Por este documento, el 164 del Archivo de la Fundación Ayala, sabemos que las sospechas de Capouya eran ciertas. Ilsa Barea no llegó nunca a terminar más que un bosquejo de traducción de una parte de Muertes de perro. Herbert Weinstock, en esta carta del 25 de febrero de 1963, escribe que: «The first thing I did was read part of the translation by Ilsa Barea, which I find entirely useless». Y Weinstock añade: «Believe me that you have all my sym-

\footnotetext{
${ }^{20}$ Carta de Emile Capouya a Francisco Ayala. Archivo Fundación Francisco Ayala, documento 163, 23 de abril de 1962.

${ }^{21}$ Carta de Emile Capouya a Francisco Ayala. Archivo Fundación Ayala, documento 162, 18 de octubre de 1962.
} 
pathy and all my admiration for your patience with regard to this translation $»^{22}$.

Como ya mencionamos anteriormente, Ignacio Soldevila Durante sugirió que en realidad Ilsa Barea publicó su traducción de Muertes de perro bajo otro nombre o que hizo de intermediaria. Hemos demostrado que no ocurrió así. Tal especulación no tiene consistencia, ya que el documento 151 del Archivo de la Fundación Ayala es una carta mecanografiada con fecha del 29 de mayo de 1963 que Joan MacLean le envía a Francisco Ayala ${ }^{23}$. Este documento va encabezado con las siguientes señas postales: Mrs. Frederick N. MacLean, Beattie Road, Rock Tavern, New York. Por su contenido se deduce que desde el 25 de febrero de 1963, momento en que Weinstock se pone en contacto con Ayala, hasta ese 29 de mayo en que se fecha la comunicación de MacLean, nuestro autor estuvo en contacto con la nueva traductora y que colaboró activamente para que se acelerase el proceso de la traducción de la novela: «Thank you for your understanding of my working idiosyncracies, and for permission to write to you in English. I note with some dismay that it has been nearly two months since I last wrote you». Y continúa haciéndole saber a Ayala que ya posee un borrador completo y terminado de la novela y que para finalizar el encargo necesita que le responda sobre cuál es el giro más apropiado para la traducción de una lista de palabras ante las que tiene dudas.

La intervención de Herbert Weinstock fue decisiva. Lo que se había dilatado durante prácticamente tres años, se resolvió en tres meses. Sin embargo no tendremos noticias del lanzamiento de la traducción de la novela hasta el día 27 de marzo de 1964. En el Archivo de la Fundación Francisco Ayala se encuentra un importante documento ${ }^{24}$, una copia de una carta que la periodista y famosa crítico de literatura española, Mildred Adams, envió a la editorial Macmillan a la atención del editor Daniel Hays. En esa carta Adams felicita a la editorial por la publicación:

I understand from Francisco Ayala that you have just published in English translation his fine and famous book «Muertes de Perro». I am delighted that you and McMillan have been so fresighted. Ayala is one of the finest of modern writers in Spanish and too little known in this country. His fame in Spain and in South American is wide and growing. I hope that the publication of his book which marks his first appearance in English may be followed by other books and that it may win for him the recognition which he so well deserves.

Esta carta demuestra que el impacto inmediato de Death as a way of life, título final que recibió Muertes de perro, fue tan favorable como importante

\footnotetext{
${ }^{22}$ Carta de Herbert Weinstock a Francisco Ayala. Archivo Fundación Francisco Ayala, documento 164, 25 de febrero de 1963.

${ }^{23}$ Carta de Joan MacLean a Francisco Ayala. Archivo Fundación Francisco Ayala, documento 151, 29 de mayo de 1963.

${ }^{24}$ Carta de Mildred Adams a Daniel Hayes. Archivo Fundación Ayala, documento 170, 27 de marzo de 1964.
} 
en la recepción de la obra por parte de la crítica especializada. Adams era una de las personalidades más destacadas del hispanismo norteamericano. En efecto, en ese momento, como explicó Orringer (1996: 9-67) en su edición de Muertes de perro, Ayala gozaba ya de una acogida muy favorable en el ámbito hispánico. Esta traducción constituía, finalmente, su presentación en el mercado literario en lengua inglesa.

\section{RECEPCIÓN INMEDIATA DE LA TRADUCCIÓN DE MUERTES DE PERRO}

Gracias a los documentos del Archivo de la Fundación Ayala podemos constatar que dicha presentación fue muy exitosa y que, como el mismo Ayala había intuido en sus cartas, tendría consecuencias fundamentales en su carrera literaria porque le podría abrir las puertas a otras publicaciones y traducciones no sólo de otras de sus obras al inglés, sino de Muertes de perro a otras lenguas, tal y como ocurrió.

Efectivamente, la recepción inmediata fue extremadamente favorable. El 12 de mayo de $1964^{25}$ aparece la primera reseña del libro en The New York Times. Se trata de un texto muy elogioso y al mismo tiempo muy bien argumentado, sin caer en ningún momento en concesiones al autor. La reseña está firmada por Charles Poore y se titula «The Way of the Usurper Is Not Very Tranquil». Dicha reseña, en nuestra opinión, es excelente porque verdaderamente brinda al lector la posibilidad de saber a) quién es Francisco Ayala, b) qué lugar ocupan sus obras dentro de la literatura hispánica, c) cuál es el alcance y la originalidad de Muertes de perro, d) cuál es la contribución de esta novela al panorama internacional literario. Poore, en vez de santificar o condenar a Ayala, ofrece de manera muy convincente y sólida un conjunto de claves de lectura que sin duda fueron determinantes del éxito posterior de la novela en inglés.

Tal éxito se puede rastrear en otro documento. Se trata de una carta fechada el 14 de mayo de 1964 con la que Virginia Patterson, directora del departamento de ventas y publicidad de la editorial, envía a Ayala una reseña publicada en el Herald Tribune Book Week, que también fue publicada al mismo tiempo en el Washington Post y el San Francisco Examiner ${ }^{26}$. No se conservan copias de estas notas críticas en la Fundación Ayala pero, por alusiones en la correspondencia posterior archivada en la Fundación, conocemos que también fueron muy elogiosas. La propia Mildred Adams escribe el 15 de mayo de 1964 a Francisco Ayala dándole la enhorabuena por la traducción de Muertes de perro:

\footnotetext{
${ }^{25}$ Poore, Ch. «The way of the usurper is not very tranquil», The New York Times. 12 de mayo de 1964, p. 22.

${ }^{26}$ Carta de Virginia H. Patterson a Francisco Ayala. Archivo Fundación Francisco Ayala. Documento 166, 17 de junio de 1964
} 
I was delighted to see in a recent issue of the New York Times that Charles Poore had read your book and liked it. Yesterday, when I lunched with Francis Brown, who edits the Sunday Book Review, he told me that he plans to be carrying a review and that it should be published either May 17th or 24th [...] I need not to tell you that for a Spanish author making his first appearance before an Englishspeaking public, [...] The reviews in the Times will be ever so good for the future publicity that your work will attract ${ }^{27}$.

No le faltaba razón a Mildred Adams. Estas reseñas resultaron contribuciones importantes para la construcción de la recepción favorable de la novela, pero sobre todo fue esencial que la primera de Poore fuera tan positiva. Ayala, conocedor de que la editorial no había pagado publicidad y del hecho de que Charles Poore tampoco mantenía una ligazón profesional con la editorial Macmillan, le escribe una carta de agradecimiento por su reseña el 15 de mayo de 1964:

I want to express my gratitude to you for your kind attention to my book «Death as a Way of Life» in the May 12 edition of The New York Times. Let me say that your criticism was the first devoted to any of my novel son the basis of English translation outside of the closely specialized circle of Hispanists in the United States. It is most gratifying to me not only because such an authoritative writer as you introduces me to the American public but above all the way you did it. I am fully aware of your adeptness in perceiving a number of important points in my work and seeing beyond the barriers imposed by the unavoidable opacities of a translated text. My appreciation is profound ${ }^{28}$.

Sin embargo, en otra carta del 27 de mayo de 1964 hallamos a un Francisco Ayala descontento con ciertos aspectos de la distribución del libro. En esta comunicación, dirigida a Lee C. Deighton, presidente de la editorial Macmillan, Ayala se queja de la deficiente primera distribución de la novela:

The book was announced for publication on May 11, 1964. The day before, May 10, the Herald Tribune carried on its Sunday's «Book Week» a fine review of Death as a Way of Life. On the following day, May 12, The New York Times, in its regular issue, printed another favourable feature review by Charles Poore. Then two weeks after, the Times again called attention to my novel in its Sunday «Book Review» with another piece written by a well-known critic of Spanish literature. And yet copies of the book were not available for purchase in any of the leading Fifth Avenue bookstores until May $20^{29}$.

La crítica de Ayala, difícilmente rebatible, no tardó en tener respuesta. El día 2 de junio de 1964 Lee C. Deighton contesta con una carta en la que le

\footnotetext{
${ }^{27}$ Carta de Mildred Adams a Francisco Ayala. Archivo Fundación Francisco Ayala, documento 169, 15 de mayo de 1964.

${ }^{28}$ Carta de Francisco Ayala a Charles Poore. Archivo Fundación Francisco Ayala, documento 170, 15 de mayo de 1964.

${ }^{29}$ Carta de Francisco Ayala a Mr. Lee C. Deighton. Archivo Fundación Francisco Ayala, documento 153, 27 de mayo de 1964.
} 
anuncia que hará llegar su queja al departamento de ventas de Macmillan y le agradece haberle hecho saber que Death as a way of life estaba sufriendo tales problemas de distribución de los que él mismo no era consciente ${ }^{30}$.

Por otro lado, unos días más tarde desde el Reino Unido y con fecha del 17 de junio de 1964, Ayala recibe una carta de la editorial inglesa Michael Joseph, firmada por William Luscombe, en la que le pide una fotografía para la contraportada del libro y unas notas biográficas ${ }^{31}$. La publicación de la novela en el Reino Unido es inminente.

Con fecha de 9 de julio de 1964 encontramos en el Archivo de la Fundación Ayala otra carta de la editorial Michael Joseph firmada por Luscombe en agradecimiento por el envío de la edición americana de Death as a way of life ${ }^{32}$. Este dato no es banal. También en el Archivo de la Fundación Ayala se halla una copia de la edición americana que aparece corregida con anotaciones manuscritas que suponemos de Joan MacLean, ya que tenemos la certeza de que no son textos autógrafos de Ayala de acuerdo con la autoridad de Manuel Gómez Ros, bibliotecario de la Fundación Ayala, que suponen modificaciones importantes del texto que luego fueron incorporadas y publicadas en la edición inglesa ${ }^{33}$.

Por su parte, desde la editorial Macmillan, Mary T. Heathcote escribirá a Ayala el 9 de julio de 1964 para explicarle que le ha llegado la carta con la queja que dirigió a Deighton y que, a su regreso de las vacaciones, se encargará personalmente del asunto ${ }^{34}$.

Ayala, al regreso a Nueva York de sus vacaciones en España, intercambia algunas cartas con William Luscombe en las que se refiere a las reseñas que se habían publicado en los Estados Unidos sobre Death as a way of life. Estas cartas son los documentos 180 y 179 del Archivo de la Fundación Ayala y datan del 29 de agosto y el 9 de septiembre de 1964 respectivamente. No ofrecen más relevancia que la del cumplimiento de diversas formalidades ${ }^{35}$.

\footnotetext{
${ }^{30}$ Carta de Mr. Lee C. Deighton a Francisco Ayala. Archivo Fundación Francisco Ayala, documento 150, 2 de junio de 1964.

${ }^{31}$ Carta de William Luscombe a Francisco Ayala. Archivo Fundación Francisco Ayala, documento 182, 17 de junio de 1964.

${ }^{32}$ Carta de William Luscombe a Francisco Ayala. Archivo Fundación Francisco Ayala, documento 181, 9 de julio de 1964.

${ }^{33}$ Aunque excede el límite acotado para este trabajo y de ello daremos cuenta en un próximo estudio, no podemos dejar de mencionar que aunque ambas ediciones, la norteamericana y la inglesa, vayan firmadas por una misma persona, Joan McLean, en realidad se trata de textos que difieren sensiblemente con un gran número de adiciones, de supresiones y de cambios. Este dato abunda en la complejidad de la historia editorial de la traducción de Muertes de perro y será objeto de futuras pesquisas.

${ }^{34}$ Carta de Mary T. Heathcote a Francisco Ayala. Archivo Fundación Francisco Ayala, documento 161, 9 de julio de 1964.

${ }^{35}$ Carta de W. Luscombe a Francisco Ayala. Archivo Fundación Francisco Ayala, documento 179, 9 de septiembre de 1964. Carta de Francisco Ayala a William Luscombe. Archivo de la Fundación Francisco Ayala, documento 180, 29 de agosto de 1964.
} 
Sin embargo, el 18 de noviembre de 1964, Ayala recibe una carta de Raleigh Trevelyan, uno de los editores de Michael Joseph ${ }^{36}$. En ella se le comunica al autor que Keith Ellis, de la Universidad de Toronto, se ha puesto en contacto con la editorial ofreciendo para su posible publicación una colección de traducciones de relatos cortos de Ayala. Raleigh escribe a Ayala con el objetivo de cerciorarse de que estos relatos están libres de copyright. Al mismo tiempo le pregunta si Macmillan está considerando su publicación.

Ayala, en una carta posterior del 24 de noviembre de 1964, contesta explicando que Keith Ellis es autor de un libro excelente sobre sus propias obras, por lo que afirma que sus traducciones poseen toda garantía. En relación a la supuesta oferta de Macmillan sobre la publicación de los relatos, Ayala realiza una consideración que demuestra que su talante cordial, mantenido en esta correspondencia que hemos estudiado a pesar de las enormes e injustas dificultades con las que tuvo que enfrentarse, no estaba reñido con una perfecta y educada rotundidad ante la negligencia:

As a matter of fact, I am free to use the copyright for this collection in any way I wish. Now, concerning your assumption that perhaps Macmillan is considering it for publication, let me inform you, most confidentially, that I don't feel inclined at all to have any more of my books published by this House, because of the very careless way in which they handled DEATH AS A WAY OF LIFE. This you can judge by the attached copies of two letters which I am enclosing for your private and exclusive use ${ }^{37}$.

No se conservan copias de esas dos cartas a las que nuestro autor hace referencia en la cita, pero imaginamos que la pésima gestión llevada a cabo por Macmillan con el asunto de Ilsa Barea y una posible respuesta insatisfactoria a la queja planteada por Ayala sobre la distribución de la novela, fueron razones poderosas y más que justificadas para abandonar la editorial. De hecho, Ayala incluso le propone, en su carta, que a partir de ahora Michael Joseph se encargue en exclusividad de la traducción de sus obras: «Consequently, if you are interested in these stories, I will be willing to grant you the copyright for the English language editions of this collection, as well as eventually other books of mine».

Death as a way of life había obtenido para entonces el efecto deseado en la carrera literaria de Ayala: sus obras ya se empezaban a conocer en el mercado anglosajón y se abría la posibilidad de nuevas traducciones. Al final de esta carta, nuestro autor celebra el hecho de que la novela sea lanzada en Inglaterra en marzo de 1965, a la vez que indica que en las mismas fechas será publicada la traducción al italiano en Milán.

\footnotetext{
${ }^{36}$ Carta de Raleigh Trevelyan a Francisco Ayala. Archivo de la Fundación Francisco Ayala. Documento 178, 18 de noviembre de 1964.

${ }^{37}$ Carta de Francisco Ayala a Raleigh Trevelyan. Archivo de la Fundación Francisco Ayala, documento 175, 24 de noviembre de 1964.
} 
El editor Raleigh Trevelyan, en una carta del 30 de noviembre de 1964, le comunica que lamenta los problemas que Ayala ha tenido con Macmillan y le informa de que espera las traducciones de Keith Ellis cuando estén listas para su consideración con el objetivo de ser publicadas ${ }^{38}$.

Posteriormente, con fecha de 31 de enero de 1965 encontramos una importante carta de Francisco Ayala a Carlos Clavería ${ }^{39}$. En ella pide a Clavería que se entreviste con Raleigh Trevelyan para que en persona pueda explorar la posibilidad de hacer algún tipo de presentación de la novela contando con la presencia del propio Ayala, que podría aprovechar un periodo vacacional en la universidad. No tenemos constancia documental de que esta propuesta tuviera éxito. Tan sólo sabemos por la carta siguiente de la serie que Raleigh y Clavería se reunieron en Londres, pero sin que se llegara a concretar nada.

El 1 de marzo de 1965 Raleigh vuelve a escribir a Ayala para comunicarle que finalmente el lanzamiento de Death as a way of life tendrá lugar el 22 de marzo $^{40}$. Además, se le informa de que la editorial ha rechazado publicar el volumen de Keith Ellis con las traducciones de sus relatos. La razón fue que «Unfortunately, one always has to think in terms of money-making in publishing, and I just couldn't see a sale for them in volume form». Sin embargo, quizás a modo de compensación, le ofrece su ayuda para publicar estos relatos en revistas literarias inglesas.

El 8 de marzo de 1965 Ayala contesta a Raleigh que entiende las razones para rechazar la publicación del volumen de relatos traducidos y le propone que si «Death as a Way of Life attains success, you might then publish a translation of my other novel, El fondo del vaso, which is somewhat connected with the former, and afterward you may perhaps consider again a volume of short stories» ${ }^{41}$.

Efectivamente, el día 22 de marzo aparece finalmente la novela ante el público del Reino Unido y ya para el 30 de marzo tenemos constancia de la primera reseña en tierras inglesas. En una carta del 30 de marzo de 1965, Trevelyan comunica a Ayala la publicación de una reseña muy positiva en el The Sunday Times ${ }^{42}$.

Aquí concluye la serie de cartas que se conservan en el Archivo de la Fundación Francisco Ayala entre nuestro autor y la editorial Michael Joseph.

${ }^{38}$ Carta de Raleigh Trevelyan a Francisco Ayala. Archivo de la Fundación Francisco Ayala. documento 177, 30 de noviembre de 1964.

${ }^{39}$ Carta de Francisco Ayala a Carlos Clavería. Archivo de la Fundación Francisco Ayala, documento 176, 31 de enero de 1965.

${ }^{40}$ Carta de Raleigh Trevelyan a Francisco Ayala. Archivo Fundación Francisco Ayala, documento 172, 1 de marzo de 1965.

${ }^{41}$ Carta de Francisco Ayala a Raleigh Trevelyan. Archivo de la Fundación Francisco Ayala, documento 173, 8 de marzo de 1965 .

${ }^{42}$ Carta de Raleigh Trevelyan a Francisco Ayala. Archivo de la Fundación Francisco Ayala, documento 174, 30 de marzo de 1965. 
Por último, la serie relacionada con la historia editorial de la traducción de Muertes de perro se cierra con una última carta que, fechada el 22 de abril de 1965, Ayala envía al presidente de la editorial Macmillan, Lee C. Deighton. En este documento Ayala vuelve a expresar su asombro por el trato que su libro había recibido desde la editorial:

I was pleased to see in last Sunday's issue of The New York Times Book Review the half-page advertisement you placed there for your translation of a book by my dear colleague and friend, Ana María Matute. However, it also increased my puzzlement about the contrastingly shabby treatment Macmillan's gave a year ago to the edition of my book Death as a Way of Life, despite the excellent critical reception it had $^{43}$.

La sinceridad de Ayala resulta abrumadora. De hecho nos consta, por lo que se afirma en la parte final de esta misma carta, que a pesar de que Deighton le expresara su compromiso para investigar cuáles habían sido los problemas de distribución que había sufrido la novela, en realidad no llevó a cabo ninguna acción ya que desde el 2 de junio de 1964 Ayala no había tenido noticias no sólo de cómo se iban a solucionar estos problemas sino tampoco de la posibilidad, anunciada por la propia editorial, de que Death as a Way of Life fuera lanzada como «paperback». Ayala concluía su carta con una pregunta: «May I know whether something has been done about the matter?». Según los documentos consultados en el Archivo de la Fundación Francisco Ayala, esta pregunta jamás obtuvo respuesta.

Además de las reseñas positivas mencionadas en páginas anteriores, y para completar el panorama de la recepción crítica inmediata a la publicación de Death as a Way of Life, debemos mencionar otras dos que fueron publicadas en los Estados Unidos y que alcanzaron una notable relevancia. La primera de ellas se publicó el 26 de mayo de 1964 en las páginas de The New York Times Book Review $^{44}$. Su autor fue el profesor de Humanidades de la Universidad de California Hugo Rodríguez Alcalá. Se trata de una reseña elogiosa, que presenta a Francisco Ayala como un novelista extraordinario cuya novela Death as a Way of Life «Acclaimed in its original Spanish as a tour de force of fictional technique, the novel aims at revealing the moral sickness of our times. The setting is Latin America, but could be anywhere; the fable is universal».

A esta reseña se añade la segunda a cargo de Emile Capouya, con el título de «A Dead Dictator Doubles Them Up» y publicada el 13 de junio de 1964 en el Saturday Review ${ }^{45}$. De nuevo se trata de una reseña muy elogiosa en la que se califica a nuestro autor en los siguientes términos: «Francisco Ayala is

\footnotetext{
${ }^{43}$ Carta de Francisco Ayala a Mr. Deighton. Archivo de la Fundación Francisco Ayala, documento 184, 22 de abril de 1965.

${ }^{44}$ Rodríguez Alcalá, H. (26 mayo 1964). «For the power-hungry, a dog's death waited». The New York Times, p. 38.

${ }^{45}$ Capouya, E. (13 junio 1964). «A dead dictator doubles them up». Saturday Review, p. 31.
} 
a living classic, and apparently still at the height of his powers. That fact, sufficiently established in the Spanish-speaking world, is now made manifest to our climatures and countrymen by the publication in English of this remarkable novel».

Estas dos reseñas principales encontraron su parangón inglés en las publicadas el 4 de abril de 1965 en el Sunday Telegraph por Isabel Quigly y, sin firma, en la titulada «Banana-State Death» publicada en The Times Literary Supplement, el 8 de abril de $1965^{46}$. Ambas son reseñas positivas, pero no ofrecen un análisis profundo y meditado de la novela. Sin duda, en este sentido, las norteamericanas fueron mucho más prolijas en detalles y más entusiastas a la hora de dar a conocer la traducción de Muertes de perro.

\section{CONCLUSIÓN}

Gracias a la reconstrucción de la historia editorial de la traducción al inglés de Muertes de perro podemos concluir que supuso un paso muy importante en la consideración de las obras de Ayala en el ámbito de la recepción crítica especializada como las de un clásico moderno ${ }^{47}$ (Amorós, 1969: 376377; García Posada, 1998: 5-17; Mainer, 1993: 9-45; Orringer, 1996: 9-67). Si bien para entonces ya Ayala era un escritor muy reconocido en el ámbito hispánico, el reconocimiento internacional y la posibilidad de la traducción de sus obras a otras lenguas tuvo un punto de apoyo esencial en la traducción de Muertes de perro al inglés, ya que facilitó que nuestro autor fuera leído en otros contextos y tradiciones literarias distintas a la hispánica.

Este artículo ha querido contribuir a una mejor comprensión de un aspecto importante que no había sido estudiado, el de la historia editorial de la traducción de la novela, lo que posibilita el hecho de que del presente trabajo nazcan dos líneas de investigación que deberán ser abordadas en nuevas exploraciones críticas de las obras de Ayala. Por un lado, es necesario el cotejo y estudio textual de las dos traducciones de Death as Way of Life publicadas en los Estados Unidos y el Reino Unido respectivamente y, por otro, este artículo encontrará continuidad en el análisis de las relaciones literarias de las diversas personalidades referidas con la documentación estudiada y su implicación en la construcción, no sólo de la inmediata recepción de la traducción

${ }^{46}$ Quigly, I. (4 abril 1965). «Lost and far from home», Sunday Telegraph.

${ }^{47}$ «Carta de Mildred Adams a Daniel Hayes». Archivo Fundación Ayala, Documento 170, 27 marzo 1964. MacDowell, E. (19 junio 1983). «About books and Authors... Fiction at Schocken». The New York Times. Poore, Ch. (12 mayo 1964). «The way of the usurper is not very tranquil». The New York Times, p. 22. Quigly, I. (4 abril 1965). «Lost and far from home», Sunday Telegraph. Rodríguez Alcalá, H. (26 mayo 1964). «For the power-hungry, a dog's death waited». The New York Times, p. 38. 
ya aquí presente, sino de la repercusión literaria y el interés que ha acaparado esta novela desde el momento de su publicación en 1954 hasta la actualidad. La labor intelectual de los desterrados y exiliados hispánicos, como lo fue Francisco Ayala, conforma todo un legado que aún se encuentra en proceso de recuperación.

\section{BIBLIOGRAFÍA CITADA}

Amorós, Andrés (1969). «Francisco Ayala: Muertes de perro», Revista de Occidente. 75, pp. 376-377.

Ayala, Francisco (1964). Death as a way of life. Joan MacLean (trad.). New York: Macmillan. Ayala, Francisco (1965). Death as a way of life. Joan MacLean (trad.). London: Michael Joseph.

García Posada, Miguel (1998). «Introducción», en Francisco Ayala, Muertes de perro. Barcelona: Círculo de Lectores, pp. 5-17.

Mainer, José-Carlos (1993). «Introducción», en Francisco Ayala, Muertes de perro. Barcelona: Vicens Vives, pp. 9-45.

Orringer, Nelson R. (1996). «Introducción», en Francisco Ayala, Muertes de perro. Madrid: Cátedra, pp. 9-67.

Soldevila Durante, Ignacio (2001). Max Aub-Francisco Ayala epistolario 1952-1972. Valencia: Fundación Max Aub.

Fecha de recepción: 8 de diciembre de 2013.

Fecha de aceptación: 9 de junio de 2014. 